\title{
Permanent Atrial Fibrillation as the Terminal Stage of a Chronic Disease: Palliative Care Needs to be Considered in Selected Patients with Markedly Impaired Quality of Life
}

\author{
Giuseppe Boriani Anna Chiara Valenti Marco Vitolo \\ Cardiology Division, Department of Biomedical, Metabolic and Neural Sciences, University of Modena and Reggio \\ Emilia, Policlinico di Modena, Modena, Italy
}

Atrial fibrillation (AF) is the most common rhythm disturbance encountered in clinical practice $[1,2]$ and has been traditionally approached by classifying the arrhythmia based on its temporal patterns (first-diagnosed, paroxysmal, persistent, long-standing persistent, and permanent AF) [3]. Permanent AF is the final result of a progressive disease, leading to a marked derangement of atrial structure and function, named atrial myopathy [4, 5], and the historically used terms of "delirium cordis," or "pulsus irregularis perpetuus" in Latin, or "arythmie complète par fibrillation auriculaire" in French, well describe this terminal, irreversible stage of the disease.

On clinical grounds, decision-making has to be individualized and has to take into account the complexity of $\mathrm{AF}$, and the severity of its presentation according to different domains. This is the basis of the recently proposed characterization of AF, targeted to facilitate decisionmaking and patient management [3]. Indeed, a key element of the clinical approach to AF patients is the consideration that permanent AF is a terminal stage, with acceptance of the arrhythmia by both the physician involved in the care process and the affected patient. The unavoidable consequence is that, apart from the prevention of stroke and systemic embolism, this stage has his- torically attracted much less interest and attention of the literature as compared to the earlier, less advanced disease stages.

Patients with permanent AF have a different clinical characterization as compared with patients with nonpermanent $\mathrm{AF}$ as summarized in the Table $1[6,7]$. It is noteworthy that, especially in terms of outcome, first-detected AF, which may commonly evolve to permanent $\mathrm{AF}$, shares many characteristics of permanent $\mathrm{AF}$ and is associated with worse outcomes as compared to paroxysmal or persistent AF [6].

The article by Jones et al. [8] reports on the results of focus groups of patients with symptomatic permanent AF, within a trial of heart rate control, the RAte control Therapy Evaluation in permanent Atrial Fibrillation (RATE-AF) trial. The authors have the merit to provide useful insights on the extent and impact of symptoms in selected patients with permanent AF as well as on the im-

Commentary on the article: Jones J., Stanbury M., Haynes S., et al. Importance and Assessment of Quality of Life in Symptomatic Permanent Atrial Fibrillation: Patient Focus Groups from the RATE-AF Trial [published online ahead of print, 2020 Aug 28]. Cardiology. 2020;110. Doi: $10.1159 / 000511048$. karger@karger.com www.karger.com/crd

(c) 2020 S. Karger AG, Base

Karger!
Giuseppe Boriani

Cardiology Division, Department of Biomedical, Metabolic and Neural Sciences University of Modena and Reggio Emilia, Policlinico di Modena IT-41125 Modena (Italy)

giuseppe.boriani@unimore.it 
Table 1. Clinical profile, comorbidities, and outcomes associated with the arrhythmia in patients with permanent AF

\begin{tabular}{ll}
\hline Variable & Permanent AF patients versus paroxysmal/persistent AF patients \\
\hline Age & Older age in permanent AF patients \\
Diabetes & More common in permanent AF patients \\
CKD & More common in permanent AF patients \\
Asymptomatic presentation & More common in permanent AF patients \\
Physical inactivity & Higher prevalence in permanent AF patients \\
Impaired quality of life & More common in subsets of permanent AF patients \\
Uncontrolled ventricular rate & Less common in permanent AF patients \\
Associated left ventricular dysfunction & More common in permanent AF patients \\
Left atrial dilatation & More common in permanent AF patients \\
CHA ${ }_{2}$ DS $S_{2}$ VASc score & Higher in permanent AF patients \\
HAS-BLED score & Higher in permanent AF patients \\
Risk of all-cause death during follow-up & Higher in permanent AF patients \\
Type of oral anticoagulants & NOACs are less commonly prescribed in permanent AF patients compared to VKA \\
\hline
\end{tabular}

AF, atrial fibrillation; NOACs, non-vitamin K oral anticoagulants; VKA, vitamin K antagonists; TIA, transient ischaemic attack.

portance of different aspects of quality of life (QoL) for better assessing well-being in this setting [8].

The focus group involved 19 patients from the United Kingdom ( 8 women, 11 men, with a mean age of 74 , range 62-82) diagnosed with AF for a mean of 5 years having symptom-related impairment in QoL, corresponding to a New York Heart Association Functional class II or higher. The focus group highlighted a lack of information received by the patients about AF as a disease in terms of course and consequences and a lack of attention to QoL in consultations, reflecting the prevalence of a physician perspective rather than a patient perspective in the approach to AF. This resulted in patient feelings characterized by frustration, isolation, and reduced confidence [8]. The marked impact of AF-related symptoms in individual patients in some cases leading to severe impairment of activities of daily living and the need to take into account the concurrent impact of comorbidities that emerge from this report stress the need for a holistic approach to care of AF patients targeted not only to stroke prevention but also to patient engagement with the aim to alleviate symptoms and improve QoL. The authors should be commended for providing this original perspective, which is fully in line with the proposal for a more advanced and integrated organization of care in this type of patients affected by AF symptoms limiting QoL. This should include initiatives for patient education, also involving nurses, on the need for an appropriate lifestyle for managing comorbidities and risk factors through physical activity and exercise, correction of obesity, reduction of alcohol use, etc. [3].
The need to alleviate patient symptoms through appropriate assessment of QoL with general and AF-specific tools should become an important component of consultations in patients with permanent AF. Importantly, an adequate evaluation of QoL may be the guidance for some decisions, for example, atrioventricular node ablation for rate control, which should fully involve the patients in the risk-benefit assessment in a patient-centred perspective which imply an open dialogue with the patient and the caregivers with improved communication skills and patient empowerment. Indeed, in elderly patients with permanent $\mathrm{AF}$ and narrow $\mathrm{QRS}$ a palliative approach to $\mathrm{AF}$ through atrioventricular (AV) junction ablation in conjunction with biventricular pacing was found superior to pharmacological therapy in reducing hospitalizations and symptoms related to heart failure, and an improvement in QoL was achieved [9].

The clinical complexity of some patients affected by permanent $\mathrm{AF}$ also has to consider the negative interaction that multiple comorbidities can have on the course of the disease, as well as on patient symptoms and QoL, with important implications in some cases on clinical decision-making $[10,11]$.

In this respect, permanent $\mathrm{AF}$ will be approached with a chronic disease that at this stage may deserve consideration for palliative care. The World Health Organization (WHO) defines palliative care as an approach that improves the QoL of patients and their families experiencing severe, life-threatening illness, with the ultimate goal to offer pain and symptom relief as well as spiritual and psychosocial support. 
In a perspective that goes beyond the field of cancer, an integrated and coordinated palliative care should be applied for managing a series of chronic illness during the course of the disease and not only at the end of life, with the aim to improve symptoms and health-related QoL [12].

In conclusion, permanent AF is the advanced stage of a chronic disease that may be associated with an important impact on patient QoL in a setting of clinical complexity that may involve a series of comorbidities and a profile of frailty requiring a perspective of holistic, patient-centred care. In selected frail patients affected by AF and other complex diseases, a clinical approach considering the role of palliative care aimed at better meeting actual patient needs and preferences and with the purpose of improving symptoms and QoL has to be the primary goal even at the time of high-tech medicine in line with the motto "There is an end to cure, there is no end to care."

\section{Conflict of Interest Statement}

G.B.: no conflicts with regard to the content of the present work; outside of this work, small speaker fee from Medtronic, Boston, Biotronik, Boehringer Ingelheim, and Bayer. A.C.V. and M.V. declare no conflicts of interest.

\section{Funding Sources}

No funding was received for this work.

\section{Author Contributions}

G.B.: conception/design/draft of the work; A.C.V.: conception/design/draft/of the work; and M.V.: conception/design/draft of the work. All the authors approved the final version of the manuscript.

\section{References}

1 Boriani G, Diemberger I, Martignani C, Biffi $\mathrm{M}$, Branzi A. The epidemiological burden of atrial fibrillation: a challenge for clinicians and health care systems. Eur Heart J. 2006; 27(8):893-4.

2 Vitolo M, Proietti M, Harrison S, Lane DA, Potpara TS, Boriani G, et al. The Euro Heart Survey and EURObservational Research Programme (EORP) in atrial fibrillation registries: contribution to epidemiology, clinical management and therapy of atrial fibrillation patients over the last 20 years. Intern Emerg Med. 2020Oct;15(7):1183-92.

3 Hindricks G, Potpara T, Dagres N, Arbelo E, Bax JJ, Blomström-Lundqvist C, et al. 2020 ESC guidelines for the diagnosis and management of atrial fibrillation developed in collaboration with the European Association of Cardio-Thoracic Surgery (EACTS) [published online ahead of print, 2020 Aug 29]. Eur Heart J. 2020; ehaa612.

4 Strano S, Toni D, Ammirati F, Sanna T, Tomaino $M$, Brignole $M$, et al. Neuro-arrhythmology: a challenging field of action and research: a review from the Task Force of Neu- ro-arrhythmology of Italian Association of Arrhythmias and Cardiac Pacing. J Cardiovasc Med. 2019;20(11):731-44.

5 Schnabel RB, Haeusler KG, Healey JS, Freedman B, Boriani G, Brachmann J, et al. Searching for atrial fibrillation poststroke: a white paper of the AF-SCREEN international collaboration. Circulation. 2019;140(22):1834-50.

6 Boriani G, Proietti M, Laroche C, Diemberger I, Popescu MI, Riahi S, et al. Changes to oral anticoagulant therapy and risk of death over a 3 -year follow-up of a contemporary cohort of European patients with atrial fibrillation final report of the EURObservational Research Programme on Atrial Fibrillation (EORP-AF) pilot general registry. Int J Cardiol. 2018;271:68-74.

7 Murin J, Naditch-Brûlé L, Brette S, Chiang CE, O'Neill J, Steg PG. Clinical characteristics, management, and control of permanent vs. nonpermanent atrial fibrillation: insights from the RealiseAF survey. PLoS One. 2014; 9(1):e86443.

8 Jones J, Stanbury M, Haynes S, Bunting KV, Lobban T, Camm AJ, et al. Importance and assessment of quality of life in symptomatic permanent atrial fibrillation: patient focus groups from the RATE-AF trial [published online ahead of print, 2020 Aug 28]. Cardiology. 2020;145(10):666-75.

9 Brignole M, Pokushalov E, Pentimalli F, Palmisano P, Chieffo E, Occhetta E, et al. A randomized controlled trial of atrioventricular junction ablation and cardiac resynchronization therapy in patients with permanent atrial fibrillation and narrow QRS. Eur Heart J. 2018;39(45):3999-4008.

10 Padula MS, D’Ambrosio GG, Tocci M, D’Amico R, Banchelli F, Angeli L, et al. Home care for heart failure: can caregiver education prevent hospital admissions? A randomized trial in primary care. J Cardiovasc Med. 2019; 20(1):30-8.

11 Corazza GR, Formagnana P, Lenti MV. Bringing complexity into clinical practice: an internistic approach. Eur J Intern Med. 2019; 61:9-14.

12 Effiong A, Effiong AI. Palliative care for the management of chronic illness: a systematic review study protocol. BMJ Open. 2012;2(3): e000899.
Permanent Atrial Fibrillation and Impaired Quality of Life 